\title{
Charge-Dependent Increase in Coherence of Synchrotron Oscillation at Injection
}

\author{
J. A. MacLachlan \\ Fermi National Accelerator Laboratory Box 500, Batavia IL 60510
}

\begin{abstract}
Because of coupled bunch instability and/or because of some unidentified mechanism, bunches from the $8 \mathrm{GeV}$ Booster accelerator at Fermilab arrive in the Main Injector synchrotron with a complicated centroid distribution in phase and energy. The currently installed broad band kicker provides a maximum of $2 \mathrm{kV}$, insufficient to remove injection errors before the oscillations would de-cohere, ignoring the influence of bunch charge. Perhaps surprisingly, for sufficient but generally modest charge, the effect of potential well distortion is to maintain bunch integrity. This talk illustrates the phenomenon for injection into the Fermilab Main Injector and offers an explanation sufficiently general to apply elsewhere.
\end{abstract}

Keywords: synchrotron oscillation, collective effects, coherence PACS: $29.27 \mathrm{Bd}$

\section{INTRODUCTION}

The broad band longitudinal damper for the Fermilab Main Injector (FMI)[1] has been developed on the basis of an observation that the injection phase error signal persists for much longer than one would expect from single particle dynamics. Figure 1 shows the phase error of a single bunch as seen by the damper pickup over $10^{4}$ turns, $111 \mathrm{~ms}$.[2] Ignoring the slow wander under the influence of the radial position loop, one sees a persistent phase oscillation, generally about $\pm 10^{\circ}$ amplitude. The natural assumption is that a bunch injected out of synchronism will smear out or filament to fill a larger effective longitudinal emittance within a few synchrotron oscillation periods. Figure 2 illustrates the phase space distribution of a $0.07 \mathrm{eVs}$ bunch in the FMI modeled with single particle equations of motion (EOM) for 111 ms, 95 synchrotron oscillation periods, after injection 15 degrees $(0.79 \mathrm{~ns})$ off the synchronous phase. Figure 3 shows the centroid phase error over this time interval. Clearly there is little useful error signal after about 20 $\mathrm{ms}$, so in the FMI there must be a force that keeps the bunch together. A force, of course, can usually be written as the gradient of a potential, so one may look at the total potential including that arising from the bunch charge to identify the focusing gradient. Although the interparticle force is repulsive (defocusing), the distorted potential well has a steep focusing gradient at the bunch edge, and this distortion moves with the bunch oscillation. In lieu of a more detailed treatment of the dynamics (planned for a longer paper), a semi-quantitative explanation is offered.

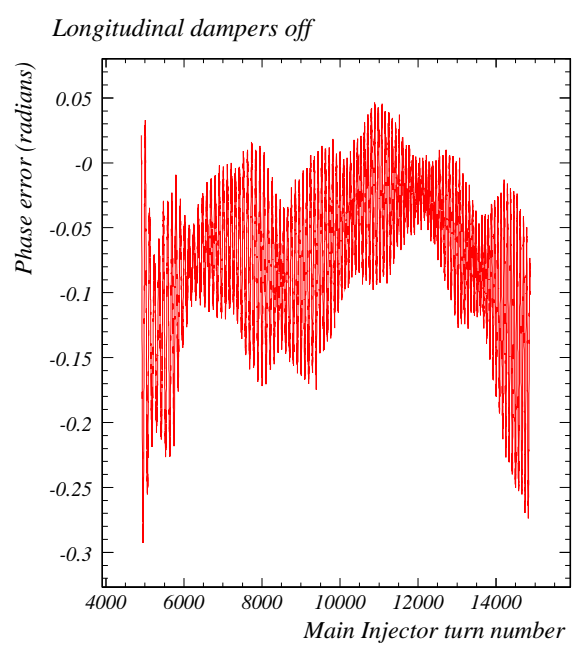

FIGURE 1. The phase of a single bunch in the FMI over 111 $\mathrm{ms}$ as seen by the longitudinal damper with no damping

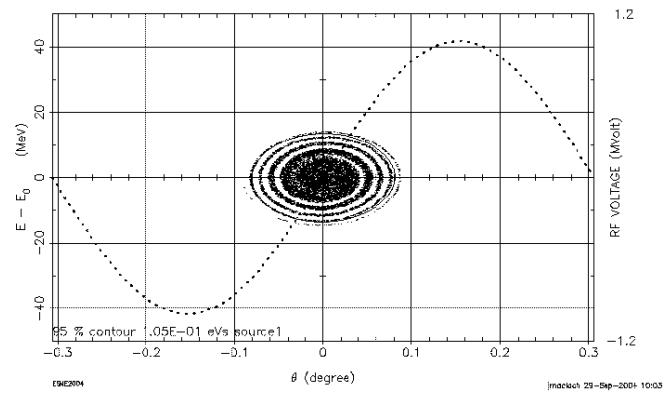

FIGURE 2. The distribution of a $0.07 \mathrm{eVs}$ bunch injected into the FMI $15^{\circ}$ off phase after $111 \mathrm{~ms}$, calculated from single particle EOM 


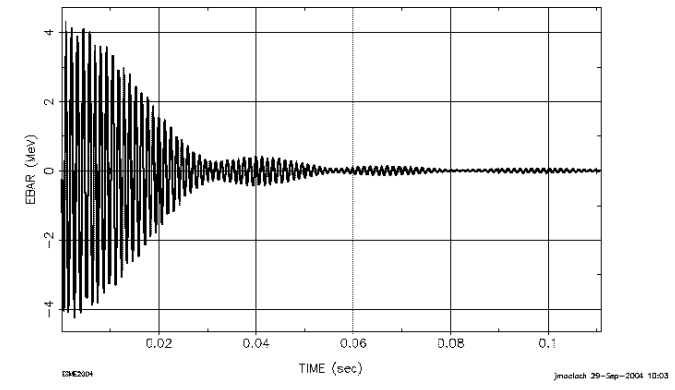

FIGURE 3. The phase error signal produced by the bunch in Fig. 2 during the $111 \mathrm{~ms}$ of filamentation of the distribution.

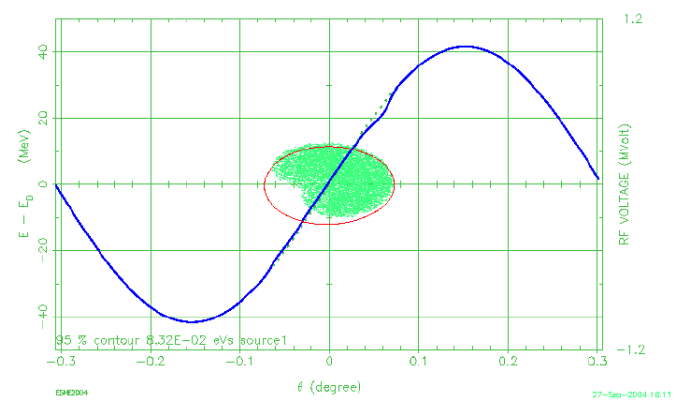

FIGURE 4. The phase space distribution of a $0.07 \mathrm{eVs}$ bunch of $6 \cdot 10^{10}$ protons injected $15^{\circ}$ off phase into the FMI after $22 \mathrm{~ms}$ (19 periods) of oscillation. The solid sinusoidal curve is the total potential, and the dotted segment indicates the unperturbed $\mathrm{rf}$ waveform.

\section{CHARGE DEPENDENT BUNCH COHERENCE}

Figures 5 and 6 are the result of tracking the evolution of a $0.07 \mathrm{eVs}$ bunch of $6 \cdot 10^{10}$ protons injected fifteen degrees off phase. The only source of longitudinal coupling is the perfectly conducting wall collective potential. A dramatic contrast between this case and the results from single particle EOM may be seen by comparing the animations

www-ap.fnal.gov/ jmaclach/movie/

MInoq_2.gif and www-ap.fnal.gov/ jmaclach/movie/MI6E10_2.gif. The former is for charge zero and the latter is under the same conditions except for a bunch charge of $6 \cdot 10^{10}$ protons. The phase error signal over the $111 \mathrm{~ms}$ in which the Fig. 5 phase space distribution evolved is given in Fig. 6; it may be compared to the single particle result shown in Fig. 3. The filamentation shown in Fig. 2 results from the dependence of synchrotron oscillation period on amplitude, that is, on the nonlinearity of the rf waveform. The sign of the space charge potential is correct to stabilize a bunch oscillating about a synchronous phase on the positive slope, i. e. , below transition. The

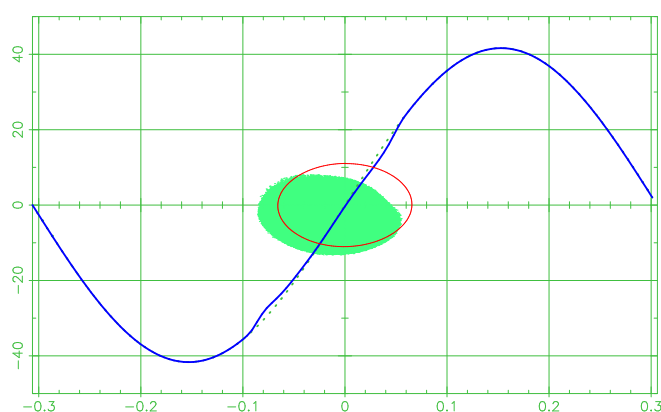

FIGURE 5. The phase space distribution of a $0.07 \mathrm{eVs}$ bunch of $6 \cdot 10^{10}$ protons injected into the FMI $15^{\circ}$ off synchronous phase after $111 \mathrm{~ms}$ (95 synchrotron oscillation periods).

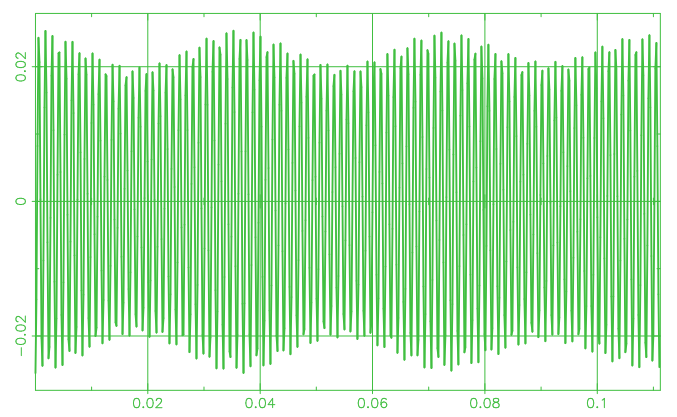

FIGURE 6. The phase error signal produced by the bunch in Fig. 5 during $111 \mathrm{~ms}$.

difference between the rf potential and a linear potential is $V_{\text {rf }}\left(\sin \phi_{\text {inj }}-\phi_{\text {inj }}\right)$, where $\phi_{\text {inj }}$ is the phase error at injection in the absence of simultaneous energy error. A necessary condition for the coherence of the bunch is that the space charge potential be sufficiently large to make up that difference. This observation allows a good estimate of the charge needed to maintain bunch coherence.

The mechanism underlying the persistence of a coherent bunch oscillation is not arcane. The perfectly conducting wall space charge force is repulsive and defocuses the incoherent oscillation of particles within the bunch; however, the effect of the potential distortion is to produce a high focusing gradient at the bunch ends and less focusing within the bunch. The incoherent oscillation frequency is reduced and for strong enough charge one expects the band of incoherent frequencies will not contain the coherent frequency at which the bunch center oscillates. When this condition is reached the so called Landau damping of the coherent oscillation is lost. This line of reasoning is now being pursued, but the numerical results are somewhat more complex than implied by the preceding assertion. The charge threshold for coherence at fixed emittance, $i$. $e$., the threshold brightness, is not sharply defined, but some coherence persists for 
thousands of turns as soon as a substantial fraction, like three quarters, for example, of the incoherent frequency band is below the coherent oscillation frequency. Until the incoherent frequencies are entirely separated, there is substantial bunch shape distortion and emittance growth, but even so the distorted bunch remains compact over thousands of turns when the coherent frequency is somewhere in the tail of the incoherent frequency band. It is planned to make this somewhat qualitative statement into a practical threshold evaluation. Success in this effort would constitute a satisfactorily fundamental understanding of the phenomenon based entirely on the basic EOM without recourse to involved analysis. Such an understanding is desirable not only because it may be easily appreciated, but because it does not raise many points requiring additional justification; it would satisfy what I have come to call Laporte's dictum: "By the time you understand it, it is trivial already."'[3] The complementary advantage of a Vlasov model is to demonstrate whether a distribution is stationary; the solution of the EOM shows this is certainly not so in the practically interesting intermediate regime wherein the coherent frequency is within the tail of the incoherent band.

\section{REMARKS}

There are several calculations, modeling results, and beam observations with a similar character of enhanced coherence of arbitrary charge distributions when the charge in that distribution is sufficiently high and the frequency distribution of the individual particles is sufficiently narrow. The physics uderlying is almost surely the same, but the initial and final distributions are disparate. Examples include the references cited.[1, 4, 5, 6]

\section{REFERENCES}

1. G. William Foster et al., "BUNCH-BY-BUNCH DIGITAL DAMPERS FOR THE FERMILAB MAIN INJECTOR AND RECYCLER”, 2003 US PAC, Portland OR (2003) http://accelconf.web.cern.ch/accelconf/p03/ PAPERS/TOPD003.PDF

2. P. Adamson, priv. comm. (2004)

3. O. Laporte, priv. comm. (1958)

4. M. Blaskiewicz, J. Wei, A. Luque, and H. Schamel "Longitudinal solitons in bunched beams", PRST-AB,7, 044402 (April 2004)

5. S. Cousineau, V. Danilov, and J. Holmes "Space-chargesustained microbunch structure in the Los Alamos Proton Storage Ring”, PRST-AB 7, 094201 (September 2004)

6. S. Koscielniak, S. Hancock, M. Lindroos "Longitudinal holes in debunched particle beams perpetuated by space-charge forces”, PRST-AB 4, 044201 (April 2001)

\section{ACKNOWLEDGMENTS}

I am grateful for the insights of Elena Shaposhnikova and Rick Baartman at this workshop which suggest a satisfying explanation of the observed coherence. Work supported by the U.S. Department of Energy under contract No. DE-AC02-76CH03000. 